\title{
Article \\ A Convolutional Neural Network-Based Method for Corn Stand Counting in the Field
}

\author{
Le Wang ${ }^{1,2}{ }^{\oplus}$, Lirong Xiang ${ }^{2}$, Lie Tang ${ }^{2}$ and Huanyu Jiang ${ }^{1, *}$ \\ 1 College of Biosystems Engineering and Food Science, Zhejiang University, 866 Yuhangtang Road, \\ Hangzhou 310058, China; wangle5994@zju.edu.cn \\ 2 Department of Agricultural and Biosystems Engineering, Iowa State University, Ames, IA 50011, USA; \\ xiang@iastate.edu (L.X.); lietang@iastate.edu (L.T.) \\ * Correspondence: hyjiang@zju.edu.cn; Tel.: +86-571-8898-2140
}

Citation: Wang, L.; Xiang, L.; Tang, L.; Jiang, H. A Convolutional Neural Network-Based Method for Corn Stand Counting in the Field. Sensors 2021, 21, 507. https://doi.org/ $10.3390 / \mathrm{s} 21020507$

Received: 15 December 2020 Accepted: 10 January 2021 Published: 13 January 2021

Publisher's Note: MDPI stays neutral with regard to jurisdictional clai$\mathrm{ms}$ in published maps and institutional affiliations.

Copyright: $@ 2021$ by the authors. Licensee MDPI, Basel, Switzerland. This article is an open access article distributed under the terms and conditions of the Creative Commons Attribution (CC BY) license (https:// creativecommons.org/licenses/by/ $4.0 /)$.

\begin{abstract}
Accurate corn stand count in the field at early season is of great interest to corn breeders and plant geneticists. However, the commonly used manual counting method is time consuming, laborious, and prone to error. Nowadays, unmanned aerial vehicles (UAV) tend to be a popular base for plant-image-collecting platforms. However, detecting corn stands in the field is a challenging task, primarily because of camera motion, leaf fluttering caused by wind, shadows of plants caused by direct sunlight, and the complex soil background. As for the UAV system, there are mainly two limitations for early seedling detection and counting. First, flying height cannot ensure a high resolution for small objects. It is especially difficult to detect early corn seedlings at around one week after planting, because the plants are small and difficult to differentiate from the background. Second, the battery life and payload of UAV systems cannot support long-duration online counting work. In this research project, we developed an automated, robust, and high-throughput method for corn stand counting based on color images extracted from video clips. A pipeline developed based on the YoloV3 network and Kalman filter was used to count corn seedlings online. The results demonstrate that our method is accurate and reliable for stand counting, achieving an accuracy of over $98 \%$ at growth stages V2 and V3 (vegetative stages with two and three visible collars) with an average frame rate of 47 frames per second (FPS). This pipeline can also be mounted easily on manned cart, tractor, or field robotic systems for online corn counting.
\end{abstract}

Keywords: deep learning; YoloV3; video tracking; corn stand counting

\section{Introduction}

Stand counting is an important task in breeding and crop management, providing farmers and breeders with information about the germination rate and population density of crops. In the breeding stage, the corn stand count can provide the seed germination rate for breeders and geneticists. As for the planting stage, stand counting can provide the plant population density to farmers, which is an important trait for crop yield and quality [1-7]. This information can also help farmers with some essential management practices, such as determining the necessity of replanting [8].

The traditional method for stand counting usually samples the corn population manually with quadrats [9], which is time consuming, laborious, and cost intensive. In addition, manual stand counts are not feasible for large fields and are prone to human error. Recent advances in machine vision provide automatic methods for farmers and breeders to count plant seedlings and determine yield-related traits such as germination rate and plant population density. One popular method has been to use a fixed camera on an unmanned aerial vehicle (UAV) to capture digital images of plants, then process the images to obtain the plant stand count offline [10-14].

UAV-based stand counting usually takes images with camera heights varying from $10 \mathrm{~m}$ to $50 \mathrm{~m}$ [11-13], with some even over $100 \mathrm{~m}[15,16]$. Thus, it is challenging to acquire 
a high-resolution image of every single plant, especially for the small plants that are in their early stages. As a result, these small objects cannot be detected accurately. Apart from UAVs, there are many ways to collect seedling images, such as moving-cart-based systems [15], tractor-based systems [17,18], and automatic field robots [19], which can collect images and information accurately and efficiently [20]. Besides RGB-camera-based methods, there are also some other sensors that can be used for object counting in the field, such as Lidar sensors [21,22].

Detecting plants accurately from images is the foundation of image-based stand counting. Different methods and their counting accuracies are summarized in Table 1. UAV-based stand counting usually requires a stitching and alignment operation. Some commercial software such as Agisoft Photoscan (Agisoft LLC, St. Petersburg, Russia) and Pixel4D (Pix4D S.A., Lausanne, Switzerland), as well as some algorithms, have been developed [11] to align UAV-collected images automatically. Then objects need to be identified from the images. In traditional image processing, different plant features have been extracted or designed to identify objects, like edge [23], color [24] and shape [12]. Some are also equipped with a hyperspectral sensor, and identify objects through NDVI index [11].

Since the significant evolution of computation in 1999, graphics processing units (GPUs) have been developed for image processing and a revolution has taken place in the application of deep learning. State-of-the-art networks include the faster region-based convolutional network (Faster R-CNN) [25], the mask region-based convolutional network (Mask-RCNN) [26], and You Only Look Once (Yolo) [27-29]. It is a promising method to develop algorithms for agricultural object detection and counting based on deep convolutional neural networks (CNNs) [13,17,30]. In particular, Faster R-CNN [25] and Yolo [28] are representative two-stage and one-stage detection networks, respectively. Each of them has made significant breakthroughs in the detection of different objects. Faster R-CNN features high accuracy, while Yolo features a high processing speed. The two networks have been widely applied in agricultural object detection. For example, DeepSeedling integrated Faster R-CNN as the target detection network, then developed a framework to track and count cotton seedlings in the videos [17]. In addition, Faster R-CNN was used as the detection network for detecting other agricultural objects such as plant leaves [31], maize seedlings [32], kiwi fruit [33], grapes [34], and apples [35]. However, most Faster-R-CNNbased approaches are offline. Real-time detection for similar objects usually implements the Yolo network [36-40], which can reach an F1 score of 0.817 for apple detection (with YoloV3) [39], and 0.944 for mango detection (with YoloV3-tiny) [36]. For this study, we chose the Yolo model to satisfy the online counting requirement.

The above image-based counting studies have achieved satisfactory counting accuracies (Table 1). For example, Rawla et al. (2018) achieved corn stand counting with an accuracy of over 99\%, using a MicaSense RedEdge Multispectral UAV Camera with a 6 $\mathrm{mm}$ lens, mounted on a Hexacopter UAV. The images were processed manually through an ImageJ-based plugin [41]. Cameras equipped with expensive hyperspectral sensors can also achieve $98 \%$ count accuracy [11]. These methods are costly, however, both in money and time.

In summary Table 1, the most commonly used counting system is UAV-based, but no real-time counting system has really been developed. The UAV-based counting systems have two major disadvantages. The first is a need for reliable hardware. A stable UAV is the basic piece of equipment, and a high-resolution camera is also needed to collect detailed information on plants from a long distance. Expensive sensors are added to improve the count accuracy. The second disadvantage is a reliance on trained experts. A trained pilot is needed to operate the UAV expertly, which is essential to the success of image acquisition and plant detection. In some methods, the image preprocessing stage also requires human intervention $[8,11,12,23,42]$. Apart from this, battery life and payload are also limitations if considering long-duration work. 
Table 1. Summary of platforms and sensing technology for stand counting in different crops.

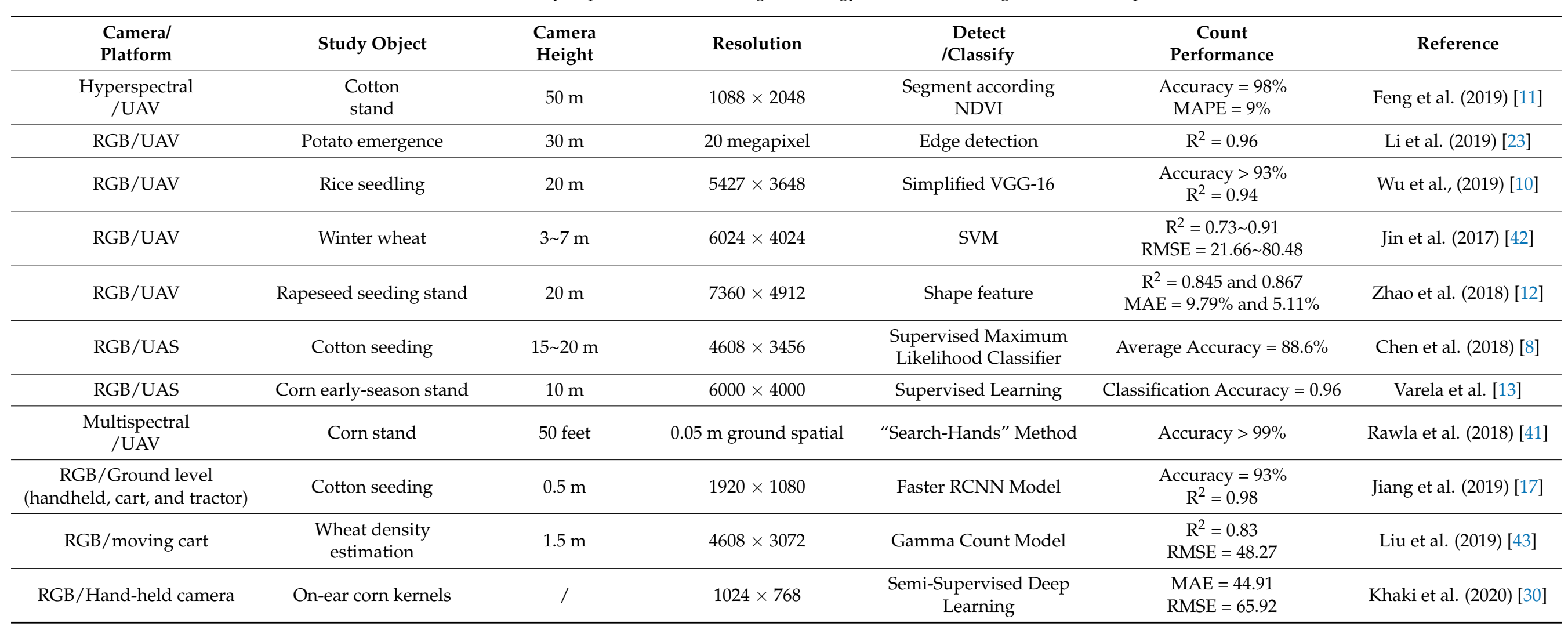


Our objective for the study was to develop a machine learning-based image processing pipeline for real-time early-season corn stand counting, which could be integrated into an unmanned robotic system for plant growth monitoring and phenotyping. This proposed corn stand counting pipeline was expected to be an accurate, efficient, and reliable solution for automatically counting early-season corn plants. No extra sensor was needed, only a camera. No professional operator was needed. The system could be easily mounted on manned cart, tractor, or field robotic systems for counting similar plants, like sorghum.

\section{Materials and Methods}

\subsection{Experimental Setting}

One week after plant emergence, video sequences were collected in corn plots planted on 2 August and 30 August 2019 at the Iowa State University Agronomy and Agricultural Engineering Research Center in Boone, Iowa (Figure 1a). Considering the uneven field, we chose a Garmin VIRB Ultra 30 action camera, with 3-axis image stabilization that captures smooth and steady video. The camera was mounted on a cart at $0.5 \mathrm{~m}$ above the ground, at the top of the corn seedlings. The camera's field of view (FOV) was $118^{\circ} \times 69.2^{\circ}$. The video was captured at 30 frames per second (FPS) with a resolution of $3840 \times 2160$ pixels. The cart was manually pushed between crop rows at about $1 \mathrm{~m} / \mathrm{s}$, with the camera looking perpendicularly down over the crop row (Figure 1b). There were 12 rows of corn plants in total.

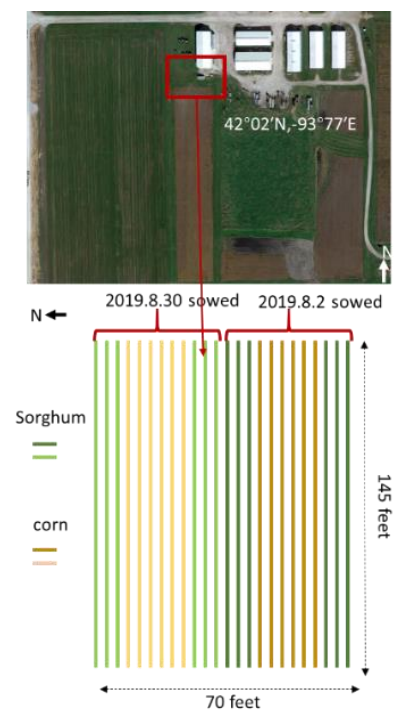

(a)

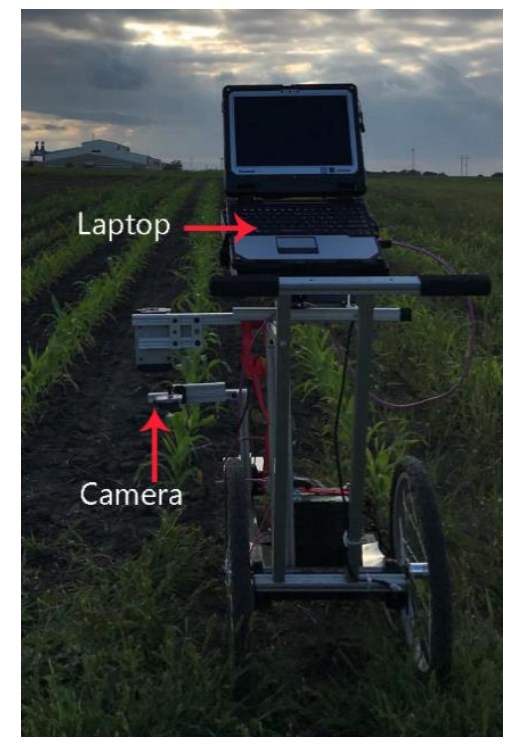

(b)

Figure 1. Diagram of the field layout and data acquisition system: (a) sketch map of sorghum and corn growth; (b) data collection cart.

According to the identification system, corn development can be divided into vegetative (V) and reproductive (R) stages [44]. The $\mathrm{V}$ stages are designated numerically as $\mathrm{V}(\mathrm{n})$, where $(\mathrm{n})$ represents the number of leaves with visible collars. We collected videos for plants from stages V1 to V4, which are the vegetative growth stages of corn plants when the first, second, third, and fourth leaf collars are visible. The row spacing of corn plants was $0.76 \mathrm{~m}$ (30 inches). The number of plants in each row was counted manually as ground-truth data.

\subsection{Image Preparation}

For training our detector model, a total of 7.2 GB of video was collected, and one image per 10 frames was extracted. There was nearly 1.5 GB per row, each of which was 145 feet. Labelme software [45] was used to draw a bounding box to annotate corn plant 
seedlings, and 864 images with corn plants at stages V1-V4 were labeled. The labeled images were split into train and validation, with a proportion of 722:142. In order to fit the detector model and improve training efficiency, the images were cropped and resized to $1024 \times 1024$ pixels. The model was trained on a desktop workstation with an Intel@ Xeon ${ }^{\circledR}$ CPU E5-2637 v3@3.5GHz × 16, and an NVIDIA TITAN GPU (Pascal) with 31.3GiB memory. For evaluation, a total of $54.9 \mathrm{~GB}$ of video was used, varying from the V1 to V4 stages.

\subsection{Corn Plant Stand Counting Pipeline}

The tracking-by-detection framework is a reliable and robust tracking method for the problem of multiple object tracking (MOT), where objects are detected in each frame and represented as bounding boxes. In this study, we developed a deep-learning-based automated pipeline to detect and count corn plant seedlings (Figure 2a). Firstly, the detection det $_{0}$ in the first frame $\mathrm{f}_{0}$ generated by YoloV3 is initialized as tracker $t r k_{0}$. In the second frame, a Kalman filter [46] is used for updating. According to the initialized bounding boxes $t r k s_{0}$, the position of objects in the second frame is predicted and denoted as $t r k s_{\text {predict }}$. Then the tracked bounding boxes are associated with bounding boxes det $_{1}$ detected in the new frame with the Hungarian algorithm. At this stage, a minimum intersection-over-union (IoU) is added to double-check the association between trk $_{\text {predict }}$ and $d e t_{1}$. If the IoU over associated trks predict $_{\text {and }} d e t_{1}$ is smaller than the pre-defined IoU

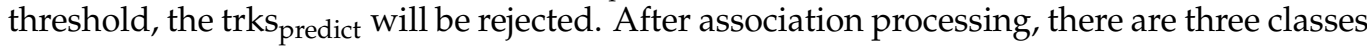
of results: trackers associated with new detection, unmatched detections, and unmatched tracking. Unmatched detections will be assigned new tracking IDs. As for the unmatched tracking, each ID owns its validity, named as max_age, which will be abandoned if it is not updated in the next few frames (total max_age number of frames). Then, the updated tracking IDs will be passed to the next epoch (Figure 2a). Finally, the counting stage is reached. We defined a finish line (the yellow line) at the bottom of the image (Figure $2 b$ ). When the trace of two tracking boxes' center points, from frame $i-1$ to frame $i$, crossed the finish line, we counted 1, shown in Figure 2b as "count".

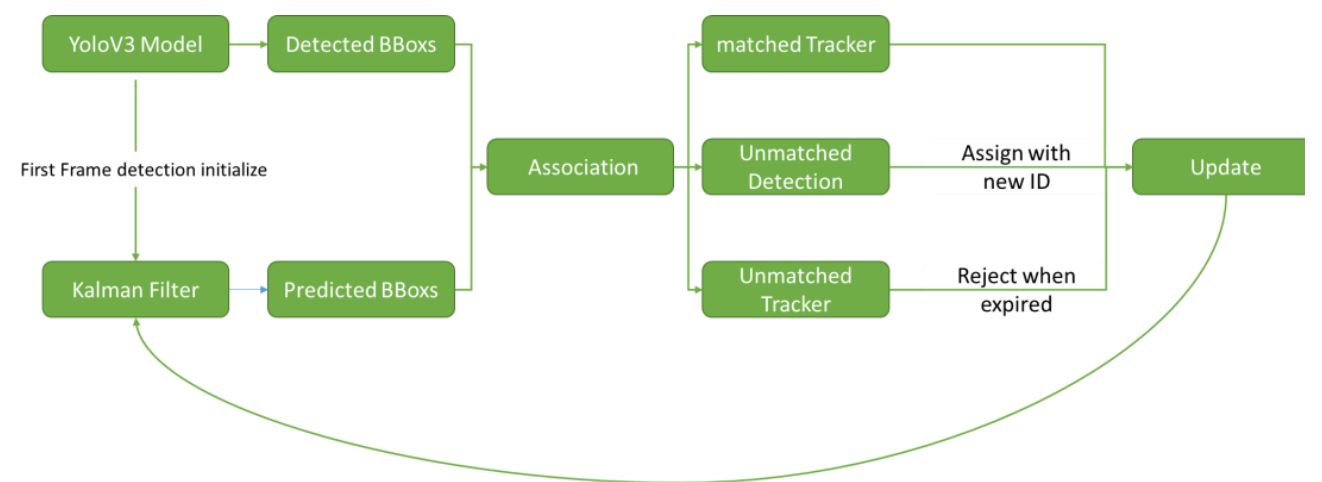

(a)
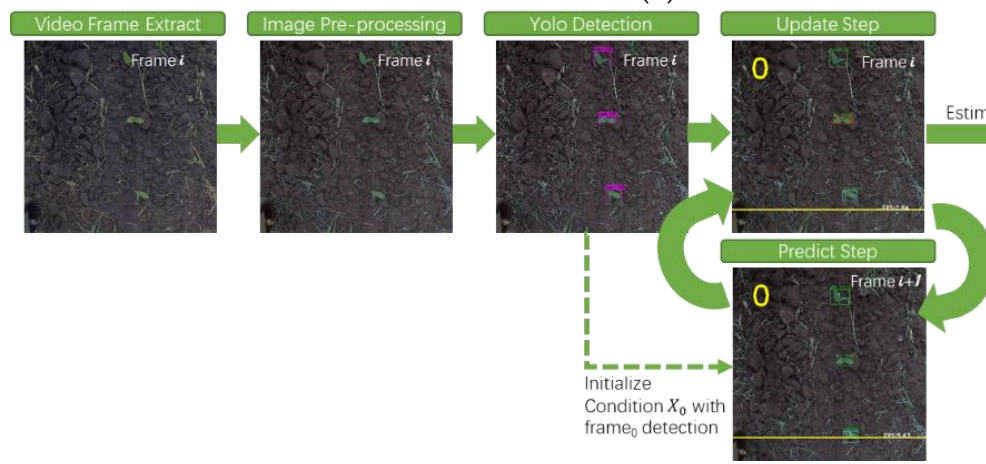

(b)

Figure 2. The counting pipeline (a) and the same shown with images (b). 


\subsubsection{YoloV3 for Corn Plant Seedling Detection}

In this study, both YoloV3 and YoloV3-tiny were trained to find the optimal model that works effectively with corn plants at various growth stages. Both of the models used Darknet [47] as the backbone network, which originally had 53 network layers trained on ImageNet [48]. Based on YoloV3, some feature layers were removed, and only two independent prediction branches have been retained for YoloV3-tiny. YoloV3-tiny has 22 layers in total, and the simplified structure results in a tiny file of only $34.7 \mathrm{MB}$, with an input resolution of $1024 \times 1024$ in this study. YoloV3 stacked 53 more layers onto the backbone, producing a 106-layer fully convolutional underlying architecture. In YoloV3, detection is carried out by applying $1 \times 1$ detection kernels on feature maps of three different sizes at three different stages in the network. The shape of the detection kernel is $1 \times 1 \times(B \times(5+C))$, where $B$ is the number of bounding boxes that a cell on the feature map can predict, " 5 " includes the four bounding box attributes and one object confidence score, and $\mathrm{C}$ is the number of classes. Taking the YoloV3 model trained on our dataset as an example (Figure 3), $\mathrm{B}=3$ and $\mathrm{C}=1$, so the kernel size is $1 \times 1 \times 18$. The feature map produced by this kernel has an identical height and width to the previous feature map [28]. YoloV3 makes predictions at three scales, which are given precisely by downsampling the dimensions of the input image by 32,16 , and 8 . On the other hand, YoloV3-tiny only keeps two feature maps for prediction, $13 \times 13$ and $26 \times 26$.
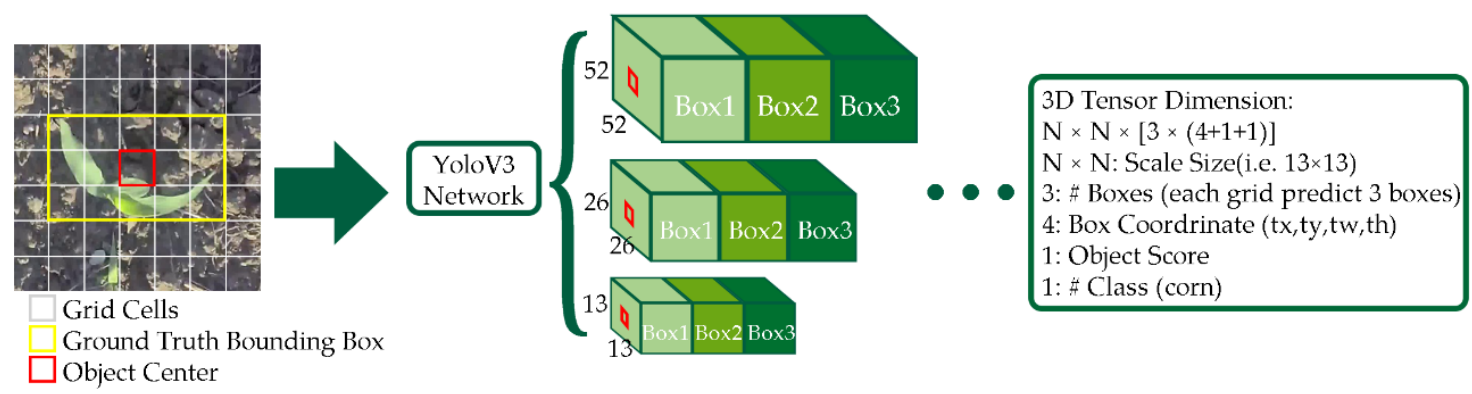

Figure 3. Prediction pipeline of a $416 \times 416$-pixel image at 3 scale sizes: $13 \times 13$ pixels, $26 \times 26$ pixels, and $52 \times 52$ pixels, with 3 bounding boxes for every cell. The grid cells are used for prediction. The red box and yellow box represent the object center and the ground truth, respectively.

\subsubsection{Corn Plant Seedling Tracking and Counting}

Once an object has been detected, it can be tracked along its path with a Kalman filter. The Kalman filter technique is a recursive solution to estimate the state of a linear system [46]. There are two main parts to this task: time update and measurement update. The time update equations are responsible for projecting the current state forward (current frame objects, detected bounding boxes) and estimating error covariance, to obtain an a priori estimate for the next frame. The measurement update equations are used to associate this estimation with the system feedback. The bounding boxes detected by YoloV3-tiny are annotated as $Z_{k}$, where the subscript $k$ indicates the discrete time. The object is to estimate a posteriori $\widehat{X_{k}}$, which is a linear combination of the priori estimate and the new measurement $Z_{k}[49,50]$.

From the network detection, we get the measurement $Z=[u, v, s, r]^{T}$. In order to predict the bounding box in the next frame, we use the following estimation model:

$$
X=[u, v, s, r, \dot{u}, \dot{v}, \dot{s}, \dot{r}]^{T}
$$

where $u$ and $v$ represent the horizontal and vertical pixel location of the target center, while $s$ represents the scale of area of the target's bounding box, and $r$ is the aspect ratio of bounding box width and height. The video was taken by a camera on a manually pushed cart moving at a roughly constant speed, so the inter-frame displacement of each object 
was approximated as a constant velocity in the model. Unlike the tracking state model for normal bounding boxes [17,51], which takes the aspect ratio $r$ as a constant, $r$ was considered as a variable that changed over time with noise in this study. The variations in $r$ can be attributed to (1) the bounding box of the same plant changing with the camera view angles, especially for the plants in V3 and V4, and (2) the aspect ratio of the same object changing because of leaf movements caused by wind, especially for stage V4. As shown in Figure 4, one individual plant was detected in consecutive video frames with different aspect ratios of the bounding box along the movement axis.
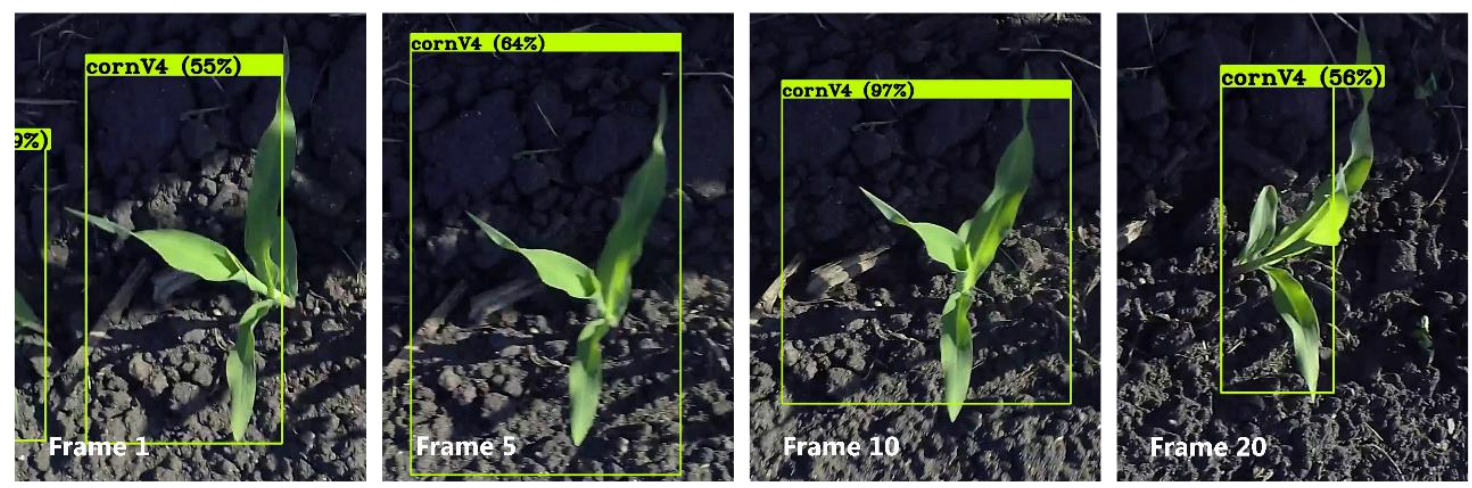

Figure 4. Example of the same object detected with different aspect ratio $r$ along the movement axis.

The usual way to count tracking objects is by counting valid tracking IDs, but the detection accuracy of YoloV3-tiny is not sufficient to satisfy the counting requirement. If the detecting network misses an object in several frames and redetects it, the old tracking ID will be abandoned and a new one will be assigned. Apart from this, the Kalman tracker is not so robust when plants appear from the field margin to the center, where the detector can detect the plant but the tracker treats it as a new object. Thus, the results of counting plants by tracking IDs will be much larger than the ground truth. Therefore, we defined a finish line CD (the yellow line in Figure 5) to count the objects. The center point A of a Tracker $i$ was connected with the center $\mathrm{B}$ of the next Tracker $I+1$. The intersection of $\mathrm{AB}$ and CD represents a valid tracking ID passing the finish line.
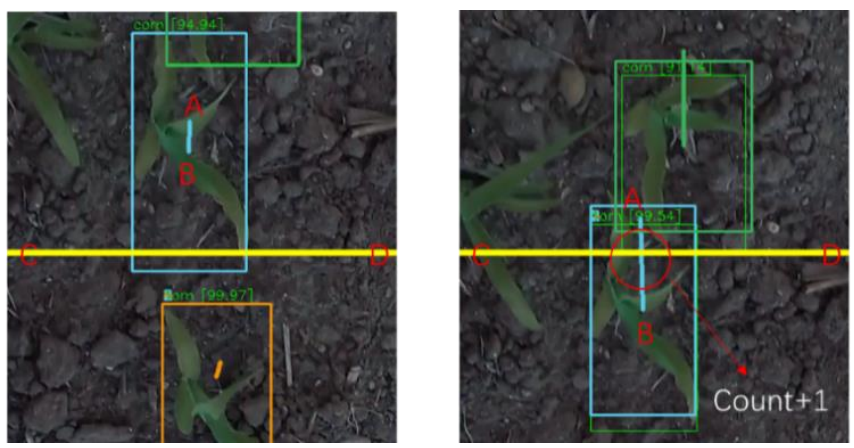

Figure 5. Demonstration of finish line for counting.

\section{Results}

\subsection{Detection}

Experiments showed that both YoloV3 and YoloV3-tiny had high mAP (mean average precision) with corn plants at growth stages V1-V4 (Figure 6). Figure 6 shows detection results with mAP@IoU 0.5 at different growth stages. The mAP@IoU 0.5 means that average precision at the IoU threshold is set as 0.5 , which means when IoU for the prediction is over 0.5 , we classify the prediction as True Positive. We compared detection performance 
with plants at stages V1-V4, and the detection results varied in different growth stages. When overlapping occurred, the detection accuracy dropped sharply.


Figure 6. YoloV3-tiny and YoloV3 detection results for four growth stages. Green = TP: true positive (object detected and matches ground-truth), red = FP: false positive (object detected but does not match ground-truth), pink = FN: false negative (object not detected but present in the ground-truth), and blue = GD: ground truth (detected object's ground-truth).

The YoloV3 and YoloV3-tiny could achieve high mAP for corn plants from the V1 to V3 stage. The highest mAP@IoU0.5 was 98.07 at stage V2 (Table 2). However, plant canopy overlapping was a major issue for large plants, where part of the whole plant was usually considered as a single object. The mAP@IOU0.5 of the V4 stage only reached 63.13 for YoloV3-tiny, and 76.33 for YoloV3. Because of this, we suggest counting from top-view images for stages V1-V3. As for the V4 stage and after, side-view image detection will be our future study plan.

Table 2. Evaluation of YoloV3 and YoloV3-tiny classification on four growth stages.

\begin{tabular}{cccccc}
\hline mAP(\%)@IoU0.5 & V1 & V2 & V3 & V4 & Average FPS \\
\hline YoloV3 & 92.78 & 98.07 & 96.94 & 76.33 & 12.21 \\
YoloV3-tiny & 89.12 & 94.13 & 93.83 & 63.13 & 76.27 \\
\hline
\end{tabular}

There was a trade-off in speed and precision. YoloV3-tiny could be an optimal solution for online counting. However, the precision needs to be improved to provide robust measurements for a Kalman filter estimator. We tried two different ways to improve the detection precision of YoloV3-tiny. The first method was to train the network to treat the four growth stages as four different classes, and the second method was to train the different stages as a sequence rather than combining them. These different training methods did improve mAP, especially for the V1 and V4 stages, and the results are shown in Figure 7.

Method 1 performed well at detecting the stages on its own. The mAP of four classes had significant improvement compared with the original YoloV3-tiny. Stage V4 reached the highest score in this method. In method 1 , it was obvious that the four growth stages appeared like three different objects. The plants at the V2 and V3 growth stages were similar to each other. In method 2, we manually weighted the stages V1 and V4 in the detection network to improve the detection mAP. Because the detected mAP was lowest for stage V4, we first trained the network on stage V4 until its loss converged, then trained stages V2 and V3 sequentially, then finally trained the V1 stage. The detecting result 
reached the best scores for stages V1, V2, and V3, which were even over $99 \%$ for stages V2 and V3. Though the training time was about four times that of the original model, method 2 was still an optimal solution due to its high accuracies.



Figure 7. The $\mathrm{mAP}(\%)$ of different training methods for YoloV3-tiny and YoloV3.

\subsection{Track and Count}

In this study, counting accuracy is defined as the formula (2). Num $G T$ is the manual seedling count number, and $\mathrm{Num}_{M}$ is the seedling number counted through our pipeline. The reason not to divide $\mathrm{Num}_{G T}$ by $\mathrm{Num}_{M}$ directly is that the pipeline may count more or less than the ground truth. So we calculate the counting accuracy through the absolute error.

$$
\text { Counting accuracy }=\left(1-\frac{\left|N u m_{G T}-N u m_{M}\right|}{N u m_{G T}}\right) \times 100 \%
$$

The count results (Figure 8) show that YoloV3-tiny can handle the detecting problem better with the customized training method, especially for stage V1. In the other growth stages, training method 2 was able to reach nearly the same count accuracy as YoloV3tiny, but it counted four times faster than YoloV3. YoloV3-tiny's average count speed was 47.1 frames per second, while YoloV3 only reached 10.7 frames per second. Aside from considering the aspect ratio changing in the state vector, we also adopted several strategies to improve the performance of the YoloV3-tiny-based counting method, including initializing the YoloV3-tiny detection model with high measurement uncertainty in a Kalman filter, and adding max age to recycle unmatched tracking IDs. We found that setting max age as 2 was best for this corn seedling counting task.

Figure 8 shows the counting result of the YoloV3 model and three method-trained YoloV3-tiny models. Our proposed method 1 and method 2 can improve significantly at the V1 stage and achieve nearly the same performance as YoloV3 at the V2 and V3 stages, while decreasing the counting accuracy standard deviation. 


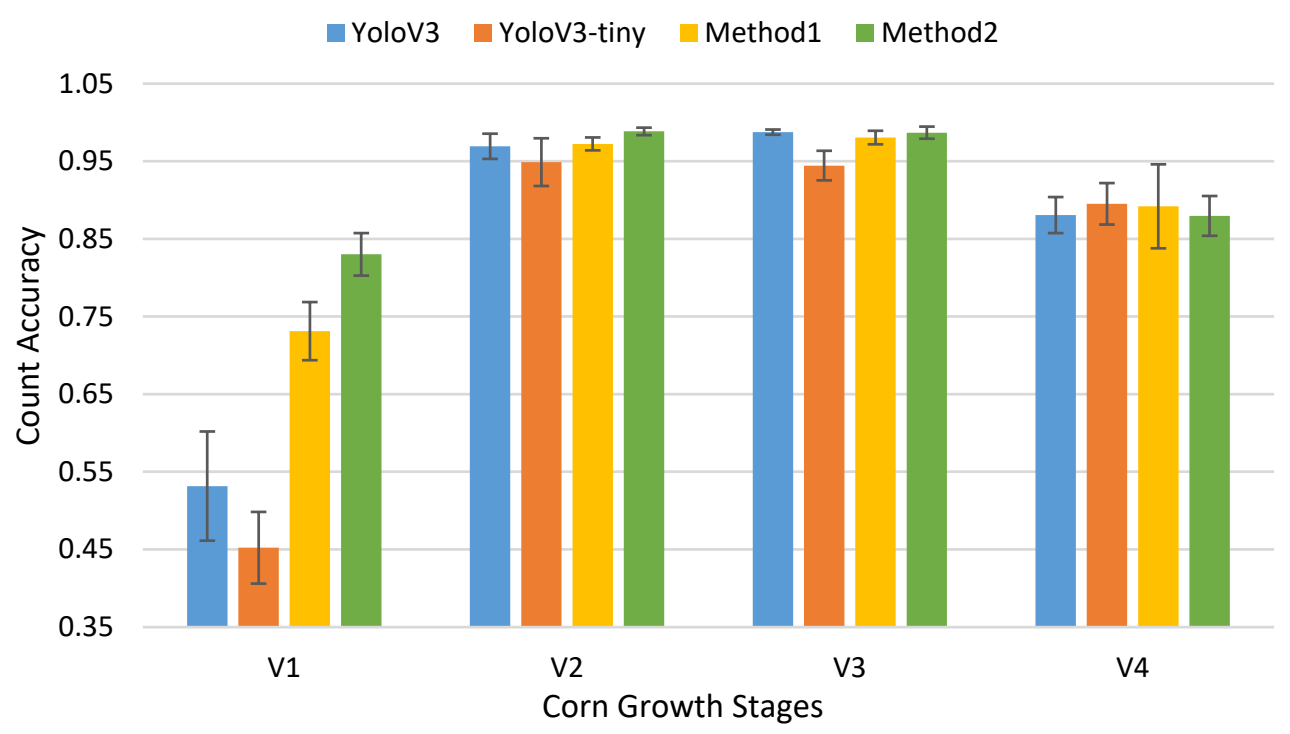

Figure 8. Count accuracy of YoloV3 and different training methods for YoloV3-tiny. The error bar represents the standard deviation of count accuracy.

\section{Discussion}

Counting for corn plants at stages V2 and V3 achieved high accuracies of $98.66 \%$ and $98.06 \%$, respectively. However, performance deteriorated when counting plants at stages V1 and V4. Detection precision for both stages V1 and V4 is lower than for the other two stages, but the main reason for this low counting accuracy is different. At stage V1, the YoloV3-tiny model can usually detect objects correctly but the Kalman filter fails to track them (Figure 9b). When detected bounding boxes are small, the values of $s$ and $r$ are small and change slightly along the time axis. In our discrete linear tracking system, the noise from motion is high as a result of the cart vibration and uneven road in the field, and this makes it difficult to track the small objects. Thus, the performance of counting is much lower than the detection. As for counting at stage V4, the counting accuracy decreases because of the overlapping and occlusions of the plant canopies. The similarity between V4, V2, and V3 will confuse the neural network. The YoloV3 model tends to detect a single corn plant as several objects (Figure 9c), and new tracking IDs are assigned to them. Thus, when they cross the finish line, the counting number is larger than the ground truth. That is the reason why counting accuracies are higher than detecting precisions when using YoloV3 and YoloV3-tiny original weight at stage V4.

Aside from the two aforementioned reasons, other random noise introduced by the detection network can also affect performance, such as missed detections (Figure 9d) and false detections (Figure 9c,e). However, these falsely detected bounding boxes generally have a very short life ( $\leq 2$ frames). It is rare that a new tracking ID will be assigned to them, and even if one is assigned, it usually expires before they cross the finish line successfully.

To improve the performance of stand counting with corn plants at the V1 stage, the camera height may need to be adjusted to obtain detailed information for tracking. If one chooses a high-resolution camera to capture images, zooming into a suitable size on the region of interest will also work (but the network must also retrain based on the old weight). In addition, there are some advanced filters which have been developed to track small targets [52-54]. As for counting plants at stage V4 and later stages, the main issue is caused by the overlap of plant canopies in top-view images. In this case, detecting plant stems in side-view images may be a better option for stand counting. 




(a)

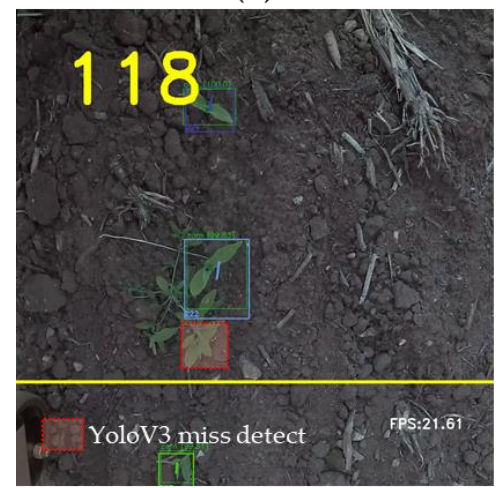

(d)

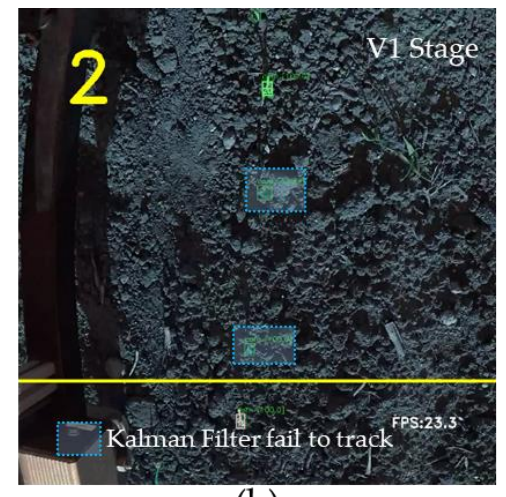

(b)

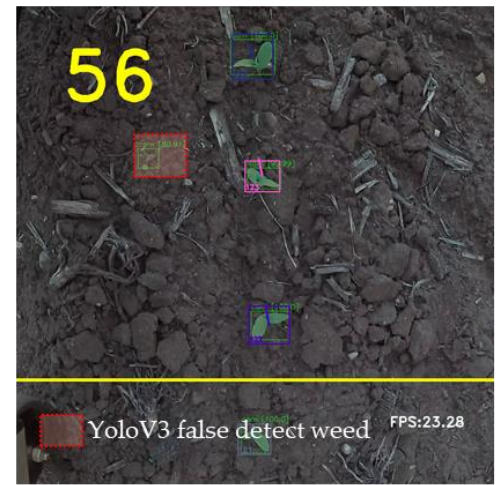

(e)

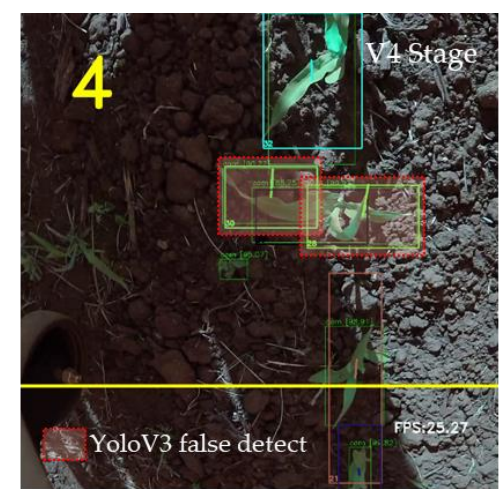

(c)

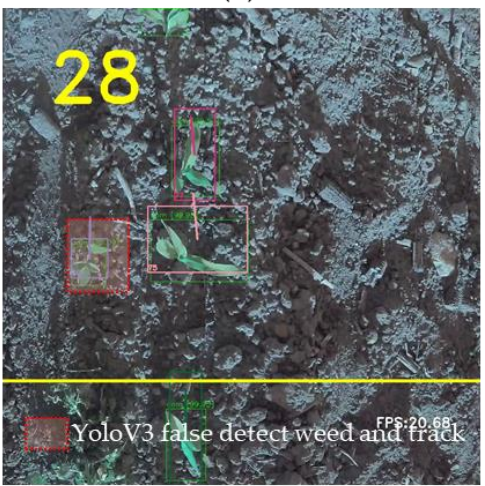

(f)

Figure 9. Summary of six different detection and tracking scenarios: (a) correctly detect and correctly track; (b) YoloV3 detects correctly but Kalman Filter fails to track; (c) detect one object as three objects and track them; (d) missed detection; (e) false detection of weed as corn; (f) false detection of weed as corn and track.

\section{Conclusions}

In this paper, we present a pipeline for counting corn seedlings in the field at growth stages from V1 to V4. The pipeline combines a powerful high-speed one-stage detection network, YoloV3-tiny, with a Kalman filter. A customized training method has also been introduced to improve YoloV3-tiny's detection accuracy. Experiments showed that this method can count corn plants at stages V2-V3 with accuracies over $98 \%$ under different outdoor lighting conditions, outperforming other CNN-based approaches for fruit counting $[10,17]$. Furthermore, the YoloV3-tiny-based model can achieve real-time counting. Thus, the pipeline is a cost-effective, high-speed, and real-time method. In addition, only one camera is needed for the system. We believe this pipeline can most likely be applied to count similar agricultural objects such as sorghum and soybean seedlings. There are still some improvements that can be made in this method, which is also our future study plan. For example, designing a height-changeable mechanical structure to suit different seedling heights could provide a big enough object (enough features) for the network to learn. A side-view of corn will be adopted to count the V4 stage and later stages, to overcome the overlapping problem.

Author Contributions: L.W. and L.T. conceived of the presented idea. All authors conceived and planned the experiments. L.W. and L.X. carried out the experiment. L.W. developed the theory and performed the computations. H.J. supervised the findings of this work. All authors discussed the results and contributed to the final manuscript. All authors have read and agreed to the published version of the manuscript.

Funding: This work was partly supported by the National Natural Science Foundation of China (grant number 31870347). 
Institutional Review Board Statement: Not applicable.

Informed Consent Statement: Not applicable.

Data Availability Statement: Data sharing not applicable.

Acknowledgments: This work was partly supported by the National Natural Science Foundation of China (grant number 31870347) and the China Scholarship Council (CSC).

Conflicts of Interest: The authors declare no conflict of interest.

\section{References}

1. Mandic, V.; Krnjaja, V.; Bijelic, Z.; Tomic, Z.; Simic, A.; Stanojkovic, A.; Petricevic, M.; Caro-Petrovic, V. The effect of crop density on yield of forage maize. Biotechnol. Anim. Husb. Biotehnol. Stoc. 2015, 31, 567-575. [CrossRef]

2. Yu, X.; Zhang, Q.; Gao, J.; Wang, Z.; Borjigin, Q.; Hu, S.; Zhang, B.; Ma, D. Planting density tolerance of high-yielding maize and the mechanisms underlying yield improvement with subsoiling and increased planting density. Agronomy 2019, 9, 370. [CrossRef]

3. Basiri, M.H.; Nadjafi, F. Effect of plant density on growth, yield and essential oil characteristics of Iranian Tarragon (Artemisia dracunculus L.) landraces. Sci. Hortic. 2019, 257, 108655. [CrossRef]

4. Zhi, X.; Han, Y.; Li, Y.; Wang, G.; Du, W.; Li, X.; Mao, S.; Feng, L. Effects of plant density on cotton yield components and quality. J. Integr. Agric. 2016, 15, 1469-1479. [CrossRef]

5. Zhang, D.; Sun, Z.; Feng, L.; Bai, W.; Yang, N.; Zhang, Z.; Du, G.; Feng, C.; Cai, Q.; Wang, Q.; et al. Maize plant density affects yield, growth and source-sink relationship of crops in maize/peanut intercropping. Field Crop. Res. 2020, 257, 107926. [CrossRef]

6. Li, X.; Han, Y.; Wang, G.; Feng, L.; Wang, Z.; Yang, B.; Du, W.; Lei, Y.; Xiong, S.; Zhi, X.; et al. Response of cotton fruit growth, intraspecific competition and yield to plant density. Eur. J. Agron. 2020, 114, 125991. [CrossRef]

7. Fischer, R.A.; Moreno Ramos, O.H.; Ortiz Monasterio, I.; Sayre, K.D. Yield response to plant density, row spacing and raised beds in low latitude spring wheat with ample soil resources: An update. Field Crop. Res. 2019, 232, 95-105. [CrossRef]

8. Chen, R.; Chu, T.; Landivar, J.A.; Yang, C.; Maeda, M.M. Monitoring cotton (Gossypium hirsutum L.) germination using ultrahighresolution UAS images. Precis. Agric. 2018, 19, 161-177. [CrossRef]

9. Fidelibus, M.W.; Mac Aller, R.T.F. Methods for Plant Sampling. Available online: http://www.sci.sdsu.edu/serg/techniques/ mfps.html (accessed on 3 July 2020).

10. Wu, J.; Yang, G.; Yang, X.; Xu, B.; Han, L.; Zhu, Y. Automatic Counting of in situ Rice Seedlings from UAV Images Based on a Deep Fully Convolutional Neural Network. Remote Sens. 2019, 11, 691. [CrossRef]

11. Feng, A.; Sudduth, K.; Vories, E.; Zhou, J. Evaluation of cotton stand count using UAV-based hyperspectral imagery. In Proceedings of the 2019 ASABE Annual International Meeting, Boston, MA, USA, 7-10 July 2019. [CrossRef]

12. Zhao, B.; Zhang, J.; Yang, C.; Zhou, G.; Ding, Y.; Shi, Y.; Zhang, D.; Xie, J.; Liao, Q. Rapeseed seedling stand counting and seeding performance evaluation at two early growth stages based on unmanned aerial vehicle imagery. Front. Plant Sci. 2018, 9, 1-17. [CrossRef]

13. Varela, S.; Dhodda, P.R.; Hsu, W.H.; Prasad, P.V.V.; Assefa, Y.; Peralta, N.R.; Griffin, T.; Sharda, A.; Ferguson, A.; Ciampitti, I.A. Early-season stand count determination in Corn via integration of imagery from unmanned aerial systems (UAS) and supervised learning techniques. Remote Sens. 2018, 10, 343. [CrossRef]

14. Burud, I.; Lange, G.; Lillemo, M.; Bleken, E.; Grimstad, L.; Johan From, P. Exploring Robots and UAVs as Phenotyping Tools in Plant Breeding. IFAC-PapersOnLine 2017, 50, 11479-11484. [CrossRef]

15. Sankaran, S.; Khot, L.R.; Carter, A.H. Field-based crop phenotyping: Multispectral aerial imaging for evaluation of winter wheat emergence and spring stand. Comput. Electron. Agric. 2015, 118, 372-379. [CrossRef]

16. Castro, W.; Junior, J.M.; Polidoro, C.; Osco, L.P.; Gonçalves, W.; Rodrigues, L.; Santos, M.; Jank, L.; Barrios, S.; Valle, C.; et al. Deep learning applied to phenotyping of biomass in forages with uav-based rgb imagery. Sensors 2020, 20, 4802. [CrossRef] [PubMed]

17. Jiang, Y.; Li, C.; Paterson, A.H.; Robertson, J.S. DeepSeedling: Deep convolutional network and Kalman filter for plant seedling detection and counting in the field. Plant Methods 2019, 15, 1-19. [CrossRef]

18. Shrestha, D.S.; Steward, B.L. Automatic corn plant population measurement using machine vision. Trans. Am. Soc. Agric. Eng. 2003, 46, 559-565. [CrossRef]

19. Kayacan, E.; Zhang, Z.; Chowdhary, G. Embedded High Precision Control and Corn Stand Counting Algorithms for an Ultra-Compact 3D Printed Field Robot. Robotics 2018. [CrossRef]

20. Li, Z.; Guo, R.; Li, M.; Chen, Y.; Li, G. A review of computer vision technologies for plant phenotyping. Comput. Electron. Agric. 2020, 176, 105672. [CrossRef]

21. Ristorto, G.; Gallo, R.; Gasparetto, A.; Scalera, L.; Vidoni, R.; Mazzetto, F. A mobile laboratory for orchard health status monitoring in precision farming. Chem. Eng. Trans. 2017, 58, 661-666. [CrossRef]

22. de Oliveira, L.T.; de Carvalho, L.M.T.; Ferreira, M.Z.; de Andrade Oliveira, T.C.; Júnior, F.W.A. Application of LIDAR to forest inventory for tree count in stands of Eucalyptus sp. Cerne 2012, 18, 175-184. [CrossRef]

23. Li, B.; Xu, X.; Han, J.; Zhang, L.; Bian, C.; Jin, L.; Liu, J. The estimation of crop emergence in potatoes by UAV RGB imagery. Plant Methods 2019, 15, 1-13. [CrossRef] [PubMed] 
24. Praveen Kumar, J.; Domnic, S. Image based leaf segmentation and counting in rosette plants. Inf. Process. Agric. 2019, 6, 233-246. [CrossRef]

25. Gavrilescu, R.; Fo, C.; Zet, C.; Cotovanu, D. Faster R-CNN: An Approach to Real-Time Object Detection. In Proceedings of the 2018 International Conference and Exposition on Electrical And Power Engineering (EPE), Iasi, Romania, 18-19 October 2018; pp. 2018-2021.

26. He, K.; Gkioxari, G.; Dollár, P.; Girshick, R. Mask R-CNN. IEEE Trans. Pattern Anal. Mach. Intell. 2020, 42, 386-397. [CrossRef] [PubMed]

27. Redmon, J.; Divvala, S.; Girshick, R.; Farhadi, A. You only look once: Unified, real-time object detection. In Proceedings of the IEEE Conference on Computer Vision and Pattern Recognition (CVPR), Las Vegas, NV, USA, 26 June-1 July 2016 ; pp. 779-788.

28. Redmon, J.; Farhadi, A. YOLOv3: An Incremental Improvement. arXiv 2018, arXiv:1804.02767.

29. Redmon, J.; Farhadi, A. YOLO9000: Better, faster, stronger. In Proceedings of the 2017 IEEE Conference on Computer Vision and Pattern Recognition (CVPR), Honolulu, HI, USA, 21-26 July 2017; pp. 6517-6525. [CrossRef]

30. Khaki, S.; Pham, H.; Han, Y.; Kuhl, A.; Kent, W.; Wang, L. DeepCorn: A Semi-Supervised Deep Learning Method for HighThroughput Image-Based Corn Kernel Counting and Yield Estimation. arXiv 2020, arXiv:2007.10521.

31. Zhu, X.; Zhu, M.; Ren, H. Method of plant leaf recognition based on improved deep convolutional neural network. Cogn. Syst. Res. 2018, 52, 223-233. [CrossRef]

32. Quan, L.; Feng, H.; Lv, Y.; Wang, Q.; Zhang, C.; Liu, J.; Yuan, Z. Maize seedling detection under different growth stages and complex field environments based on an improved Faster R-CNN. Biosyst. Eng. 2019, 184, 1-23. [CrossRef]

33. Fu, L.; Feng, Y.; Majeed, Y.; Zhang, X.; Zhang, J.; Karkee, M.; Zhang, Q. Kiwifruit detection in field images using Faster R-CNN with ZFNet. IFAC-PapersOnLine 2018, 51, 45-50. [CrossRef]

34. Santos, T.T.; de Souza, L.L.; dos Santos, A.A.; Avila, S. Grape detection, segmentation, and tracking using deep neural networks and three-dimensional association. Comput. Electron. Agric. 2020, 170, 105247. [CrossRef]

35. Fu, L.; Majeed, Y.; Zhang, X.; Karkee, M.; Zhang, Q. Faster R-CNN-based apple detection in dense-foliage fruiting-wall trees using RGB and depth features for robotic harvesting. Biosyst. Eng. 2020, 197, 245-256. [CrossRef]

36. Shi, R.; Li, T.; Yamaguchi, Y. An attribution-based pruning method for real-time mango detection with YOLO network. Comput. Electron. Agric. 2020, 169, 105214. [CrossRef]

37. Reis, M.J.C.S.; Morais, R.; Peres, E.; Pereira, C.; Contente, O.; Soares, S.; Valente, A.; Baptista, J.; Ferreira, P.J.S.G.; Bulas Cruz, J. Automatic detection of bunches of grapes in natural environment from color images. J. Appl. Log. 2012, 10, 285-290. [CrossRef]

38. Liu, G.; Nouaze, J.C.; Mbouembe, P.L.T.; Kim, J.H. YOLO-tomato: A robust algorithm for tomato detection based on YOLOv3 Sensors 2020, 20, 2145. [CrossRef] [PubMed]

39. Tian, Y.; Yang, G.; Wang, Z.; Wang, H.; Li, E.; Liang, Z. Apple detection during different growth stages in orchards using the improved YOLO-V3 model. Comput. Electron. Agric. 2019, 157, 417-426. [CrossRef]

40. Fu, L.; Feng, Y.; Wu, J.; Liu, Z.; Gao, F.; Majeed, Y.; Al-Mallahi, A.; Zhang, Q.; Li, R.; Cui, Y. Fast and accurate detection of kiwifruit in orchard using improved YOLOv3-tiny model. Precis. Agric. 2020. [CrossRef]

41. Rawla, P.; Sunkara, T.; Gaduputi, V.; Jue, T.L.; Sharaf, R.N.; Appalaneni, V.; Anderson, M.A.; Ben-Menachem, T.; Decker, G.A.; Fanelli, R.D.; et al. Plant-stand Count and Weed Identification Mapping Using Unmanned Aerial Vehicle Images. Gastrointest. Endosc. 2018, 10, 279-288. [CrossRef]

42. Jin, X.; Liu, S.; Baret, F.; Hemerlé, M.; Comar, A. Estimates of plant density of wheat crops at emergence from very low altitude UAV imagery. Remote Sens. Environ. 2017, 198, 105-114. [CrossRef]

43. Liu, S.; Baret, F.; Allard, D.; Jin, X.; Andrieu, B.; Burger, P.; Hemmerlé, M.; Comar, A. A method to estimate plant density and plant spacing heterogeneity: Application to wheat crops. Plant Methods 2017, 13, 1-11. [CrossRef]

44. Abendroth, L.J.; Elomere, R.W.; Boyer, M.J.; Marlay, S.K. Corn Growth and Development; Iowa State Univ.: Ames, IA, USA, 2011.

45. Torralba, A.; Russell, B.C.; Yuen, J. LabelMe: Online image annotation and applications. Proc. IEEE 2010, 98, 1467-1484. [CrossRef]

46. Kalman, R.E. A new approach to linear filtering and prediction problems. J. Fluids Eng. Trans. ASME 1960, 82, 35-45. [CrossRef]

47. Redmon, J. Darknet. Available online: http:/ / pjreddie.com/darknet/ (accessed on 3 May 2020).

48. Russakovsky, O.; Deng, J.; Su, H.; Krause, J.; Satheesh, S.; Ma, S.; Huang, Z.; Karpathy, A.; Khosla, A.; Bernstein, M.; et al. ImageNet Large Scale Visual Recognition Challenge. Int. J. Comput. Vis. 2015, 115, 211-252. [CrossRef]

49. Li, X.; Wang, K.; Wang, W.; Li, Y. A multiple object tracking method using Kalman filter. In Proceedings of the 2010 IEEE International Conference on Information and Automation, Harbin, China, 20-23 June 2010; pp. 1862-1866. [CrossRef]

50. Patel, H.A.; Thakore, D.G. Moving Object Tracking Using Kalman Filter. Int. J. Comput. Sci. Mob. Comput. $2013,2,326-332$.

51. Wojke, N.; Bewley, A.; Paulus, D. Simple online and realtime tracking with a deep association metric. In Proceedings of the 2017 IEEE International Conference on Image Processing (ICIP), Beijing, China, 17-20 September 2017; pp. 3645-3649. [CrossRef]

52. Davies, D.; Palmer, P.L.; Mirmehdi, M. Detection and tracking of very small low-contrast objects. In Proceedings of the British Machine Vision Conference 1998, BMVC 1998, Southampton, UK, January 1998. [CrossRef]

53. Blostein, S.; Huang, T. Detecting small, moving objects in image sequences using sequen- tial hypothesis testing. IEEE Trans. Signal Process. 1991, 39, 1611-1629. [CrossRef]

54. Ffrench, P.A.; Zeidler, J.R.; Ku, W.H. Enhanced detectability ofsmall objects in correlated clutter using an improved 2-d adaptive lattice algorithm. IEEE Trans. IP 1997, 6, 383-397. 